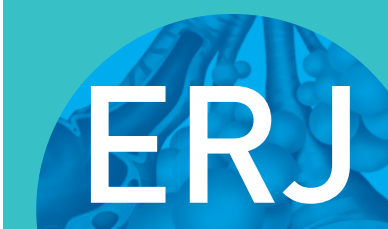

open research
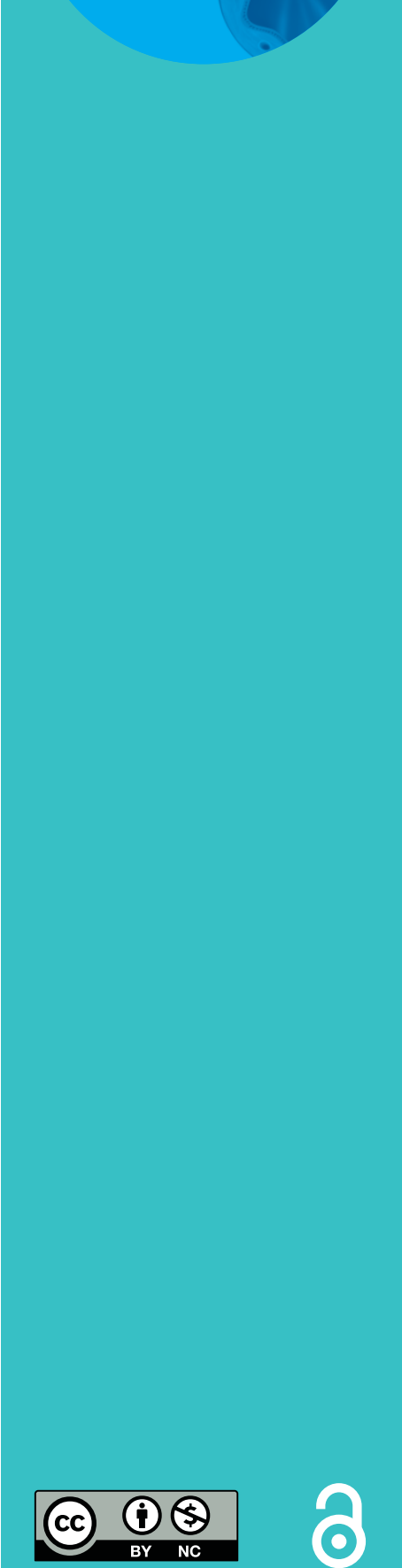

\section{ERS International Congress 2020: highlights from the Thoracic Surgery and Transplantation Assembly}

\author{
Dorina Esendagli (1) 1 , Unmil Shah², Guntug Batihan (1) ${ }^{3}$, Dimitrios Magouliotis ${ }^{4}$, \\ Federica Meloni $\mathbb{1}^{5}$, Robin Vos $\mathbb{1}^{6}$, Stefano Elia ${ }^{7}$ and Merel Hellemons ${ }^{8}$
}

Affiliations: ${ }^{1}$ Chest Diseases Dept, Baskent University Hospital, Ankara, Turkey. ${ }^{2}$ Heart and Lung Transplant Institute, KIMS, Telangana and Global Hospital, Mumbai, India. ${ }^{3}$ Dept of Thoracic Surgery, Dr Suat Seren Chest Disease and Chest Surgery Education and Research Center, Izmir, Turkey. ${ }^{4}$ Dept of Thoracic and Cardiovascular Surgery, University of Thessaly, Larissa, Greece. ${ }^{5}$ Dept of Respiratory Diseases, University and IRCCS San Matteo Foundation, Pavia, Italy. ${ }^{6}$ Dept of Respiratory Diseases, University Hospitals Leuven and Dept CHROMETA, BREATHE, KU Leuven, Leuven, Belgium. ${ }^{7}$ Dept of Thoracic Surgery, Tor Vergata University, Rome, Italy. ${ }^{8}$ Dept of Pulmonary Medicine, Division of lung Transplantation, Erasmus Medical Center, Rotterdam, The Netherlands.

Correspondence: Merel Hellemons, Dept of Pulmonary Medicine, Division of Lung Transplantation, Erasmus Medical Center, Dr Molewaterplein 40, 3015GD Rotterdam, The Netherlands. E-mail: m.hellemons erasmusmc.nl

ABSTRACT The Thoracic Surgery and Lung Transplantation Assembly of the European Respiratory Society is delighted to present the highlights from the 2020 Virtual International Congress. We have selected four sessions that discussed recent advances in a wide range of topics. From the use of robotic surgery in thoracic surgery and extracorporeal life support as a bridge to lung transplantation, to lung transplantation in the era of new drugs. The sessions are summarised by early career members in close collaboration with the assembly leadership. We aim to give the reader an update on the highlights of the conference in the fields of thoracic surgery and lung transplantation.

@ERSpublications

The first "virtual" \#ERSCongress was a great success, with very diverse and important sessions on innovation and the state of the art in thoracic surgery and lung transplantation, summarised in this article https://bit.ly/392uwUA

Cite this article as: Esendagli D, Shah U, Batihan G, et al. ERS International Congress 2020: highlights from the Thoracic Surgery and Transplantation Assembly. ERJ Open Res 2021; 7: 007432020 [https://doi.org/10.1183/23120541.00743-2020].

( T) The authors 2021. This version is distributed under the terms of the Creative Commons Attribution Non-Commercial Licence 4.0. For commercial reproduction rights and permissions contact permissions@ersnet.org 


\section{Introduction}

Assembly 8, Thoracic Surgery and Transplantation, includes physicians and surgeons with an extraordinary knowledge of the state of the art in the field of thoracic surgery (Group 8.1) and lung transplantation (Group 8.2). Both groups are strongly engaged with the other Scientific Assemblies, focussing on surgical options for the treatment of lung diseases. The overall number of members, as of August 31,2020, is about 500 with a steady yearly increase of $10 \%$. The rate of acceptance of abstracts at the ERS International Congress is very high $(86.5 \%$ in Vienna) thus indicating a high quality of submitted abstracts.

The members of Thoracic Surgery Group include surgeons who have a special interest in an interdisciplinary approach towards various thoracic pathologies eventually requiring surgical intervention in terms of a diagnostic or a therapeutic procedure, such as malignancies, pleuro-pulmonary or mediastinal infections, trauma or other benign diseases of the lung, pleura or mediastinum. The group focusses on the possibilities of interdisciplinary and interactive sessions during the ERS International Congress in order to create a culture of interdisciplinary between surgical and non-surgical members of the Society.

The members of Transplantation Group include pulmonologists/respiratory specialists who have a particular interest in lung transplantation. It is a group strongly engaged in collaborative working to better understand the risk factors, mechanisms and treatment options for lung transplant recipients who develop chronic lung allograft dysfunction affecting their post-transplant survival and causing post-transplant morbidity. This group focusses on high-quality symposia during the annual congress, it is publishing monographs on Lung transplantation, it already delivered three very successful ERS School Courses and it organised several Meet the Professor sessions.

In the last congress, Assembly 8 organised/took part in two symposia, two joint sessions with European Society of Thoracic Surgeons, one pro/con debate, one expert view. The highlights of these important sessions are represented in this paper.

\section{Group 8.1 Thoracic surgery}

Pro/Con debate: Do I need a robot in my thoracic surgery operation theater?

This session debated the potential utility of robotic technology for thoracic surgery.

\section{Pro: Progress in robot-assisted thoracic surgery (RATS)}

In her presentation, Giulia Veronesi provided all the recent evidence on the progress and utility of robot implementation in the field of thoracic surgery. After demonstrating the evolution from open surgery to minimally invasive, robotic and digital surgery, she pointed to the great possibilities that big data and machine learning bring to thoracic surgery by providing increased possibilities of preoperative surgical planning and enhancing outcomes. In this context, the adoption of robotic technology was rapid in recent years, with the number of robot-assisted lobectomies reaching up to $18 \%$ in 2016 , from $1 \%$ in 2010 . In addition, she stressed the technical advantages that the robotic approach provides, such as intuitive movements, tremor filtration, increased degrees of freedom, motion scaling, stereoscopic vision and eye-hand-target alignment. Due to these advantages, RATS is associated with a learning curve of 20 patients in contrast to the over 50 patients that are needed for video-assisted thoracoscopic surgery (VATS) [1]. All these characteristics contribute to enhanced clinical outcomes, in terms of readmissions and grade 3-4 complications. In fact, RATS is associated with lower mortality rate compared with open surgery and VATS, improved quality of life and reduced pain compared to open surgery, improved lymph node dissection and radicality regarding either anatomic segmentectomy [2] or a more extended resection for locally advanced non-small cell lung cancer [3] compared with VATS, with similar oncological outcomes. Furthermore, RATS provides enhanced outcomes when applied in complex cases and thymectomy in myasthenic patients [4]. Finally, it was demonstrated that both VATS and RATS are associated with similar costs when complications, length of stay and readmissions are taken into consideration.

\section{Con: VATS superiority over RATS}

In his presentation, Rene Horsleben Petersen focussed on three topics: 1) advantages and disadvantages of RATS; 2) advantages of VATS; and 3) the potential of robotic systems evolution in the near future. Petersen listed the disadvantages of RATS as they were presented in a recent review [5]. These disadvantages included higher cost, longer operation time, the necessity of a skilled assistant on the table, the lack of tactile feedback, along with the number and size of the ports [5]. Petersen then demonstrated newly developed equipment for VATS that is not available in RATS, such as advanced staplers and energy systems. Besides, the introduction of three dimensional and uniportal VATS has increased the feasibility and ergonometric of VATS at levels equal to RATS. According to the outcomes of a meta-analysis that 
compared VATS and RATS, no significant difference was reported regarding conversion to open rate, number of dissected lymph nodes, length of hospital stay, mean operation time, drainage volume after surgery, and postoperative morbidity [6]. Nonetheless, that meta-analysis was associated with certain limitations, such as high heterogeneity in pooled outcomes and not clearly indicated inclusion-exclusion criteria regarding the stage of disease and the extent of surgery, thus posing biases in its conclusions [6]. For final positioning of RATS future robotic developments should be awaited. Furthermore, there is important potential of artificial intelligence, big data, image fusion and other novelties in thoracic surgery.

\section{Highlights}

- The number of robot-assisted lobectomies has increased tremendously.

- It remains unclear whether the advantages of robot-assisted thoracic surgery outweigh the disadvantages, especially in the light of other innovations in the field of VATS.

\section{What's new with ground glass opacities?}

Increased use of chest computed tomography in daily practice not only provides earlier detection of lung cancer but also increased detection of incidental findings such as ground glass opacities (GGOs). This has warranted the development of specific approaches regarding follow-up, diagnosis and treatment for these lesions. In this session the management of GGOs was discussed, aiming for the optimal approach.

First, Klaus Loureiro Irion elaborated on the terminology of GGO and ground glass nodule (GGN), technical factors affecting GGN analysis, diagnosis, follow-up and biopsy recommendations. Lesions can be subdivided in pure ground glass nodules (pGGN), including AAH (atypical adenomatous hyperplasia) and AIS (adenocarcinoma in situ) and partly solid ground glass nodule (psGGN) including minimally invasive adenocarcinoma (MIA) and invasive adenocarcinoma. The larger the size of the nodule and the shorter the volume doubling time, the higher is the prevalence of malignancy [7]. If present, the solid component of the nodule should be assessed in various settings, not alone in the mediastinal setting, as the size can be underestimated. Temporal progression should be monitored and underestimated, as some GGNs don't change over the years. It's recommended doing 6-12 monthly follow-up scan for pGGN, and 3-6 monthly follow-up scans for psGGN, with a minimum of 5 years follow-up. A change in GGN size more than $2 \mathrm{~mm}$ and increased density will require evaluation [8]. Even with a negative biopsy, patients cannot be reassured, and a positive lung biopsy does not eliminate the need for lymph node staging. Individual counselling regarding risks and benefits is the key, and multidisciplinary team discussion can guide diagnostic and therapeutic decisions.

Secondly, François Montagne spoke about the role of artificial intelligence methods for the pulmonary nodule classification. Deep neural networks are designed for lung cancer classification [9] as well as different deep learning models for detecting or classifying pulmonary nodules are suggested. Currently, these models are only supportive in the clinical decision-making process. These techniques hold promise for the future.

Thirdly, Jean-Marc Baste spoke about the problems and solutions in contemporary thoracic surgery for GGO. He focussed on three basic goals in his presentation: minimally invasive sub-lobar resections, preand peri-operative visible dye marking and surgical planning using multi-images strategies. Decision making on minimally invasive surgical resection type is essential. While wedge resection is adequate for clinical stage 1 GGO with solid component $<\% 50$, segmentectomy with lymphadenectomy is suggested for clinical stage 1 GGO with solid component $>\% 50$. If lymph nodes are suspected, peri-operative frozen sections can be performed during surgery to proceed to lobectomy if positive.

Current techniques to assist in minimally invasive surgery are pre-operative planning using 3D reconstruction, real-time peroperative computed tomography scans on hybrid operation theatres or peroperative aid by bronchoscopy, as is electromagnetic navigation bronchoscopy-guided dye marking for resection of pulmonary nodules. An important technical consideration was described using Endo GIA Black Reload for the larger wedge and extended segmentectomy to increase the surgical margin.

A controversial issue is whether or not to perform lymphadenectomy for GGOs. The current state of the evidence is that for pure GGOs, there is no need for lymphadenectomy. For semi-solid GGOs lymphadenectomy is a must. The utility of sentinel node identification and of peri-operative frozen section is still to be elucidated.

Regarding pre- and peri-operative marking techniques for GGOs, the pleural dye marking using radial endobronchial ultrasound and virtual bronchoscopy was emphasised the most. A well-managed case was presented of wide wedge resection after pleural dye marking using radial endobronchial ultrasound. By presenting another case that underwent posterior (S2) segmentectomy, he emphasised that marking 
techniques are also useful in clarifying segmental plans. He concluded his presentation by emphasising the efficacy of multi-image assistance and stating that these procedures will become routine in the future.

In the final presentation of this session, Isabelle Opitz spoke about non-surgical alternatives for the treatment of GGOs. She focussed on stereotactic body radiation therapy (SBRT) and ablation methods, which include radiofrequency ablation (RFA), microwave ablation (MWA), and cryoablation (CRA).

Lung RFA of GGOs was shown to be a feasible, safe and useful therapeutic option to control GGO-dominant lung adenocarcinoma, with overall and cancer-specific survival rates $100 \%$ and $100 \%$ at 1 year, $96.4 \%$ and $100 \%$ at 3 years, and $96.4 \%$ and $100 \%$ at 5 years respectively [10]. SBRT in patients with lung tumours composed of mainly GGO yielded cause-specific and overall survival at 3 years of 98.2 and $94.6 \%$, respectively [11]. Despite these promising results, limitations of these non-surgical techniques are lack of long-term survival information, inability for pathological confirmation (for SBRT) and lymph node dissection, and difficulties in the follow-up.

In conclusion, while developing technology requires us to make new definitions like GGO, pGGN and psGGN, it helps us to improve our diagnosis and treatment methods. This session has made an important contribution to the congress by sharing the most up-to-date information and experiences in approaching GGOs.

\section{Highlights}

- Novel techniques (such as three-deimensional reconstruction and pleural dye techniques) have made minimally invasive sub-lobar resection for GGOs safe and feasible.

- There are several non-surgical alternatives for the treatment of GGOs including SBRT, RFA, MWA and CRA with good outcomes but also important disadvantages.

\section{Group 8.2: Lung transplantation}

Extracorporeal life support (ECLS) in lung failure and lung transplant bridging: when and how?

Arne Neyrick gave an overview of the technical aspects regarding ECLS and its use in severe acute respiratory failure, ARDS, and its use for bridging to lung transplantation. The use of ECLS, also named extra-corporeal membrane oxygenation (ECMO), dramatically increased in the last decade [12]. When ECMO was initially developed, it was mainly applied as a rescue strategy. It is now increasingly used in combination with other treatment strategies, such as ultraprotective ventilation or even as a scenario in which mechanical ventilation can be avoided or weaned completely. The main aim of ECMO is to improve oxygen delivery. In case of respiratory failure usually veno-venous (VV) setup is used for this purpose. In this setup there is venous withdrawal of blood, followed by gas exchange and then venous return [13]. The oxygenation capacity of this setup depends on blood flow through the system and the sweep gas fraction inspired $\mathrm{O}_{2}\left(F_{\mathrm{iO}_{2}}\right)$. Also, $\mathrm{CO}_{2}$ can be removed by this setup if applicable, and the capacity for $\mathrm{CO}_{2}$ removal depends on the gasflow. The purpose of ECMO is to optimise gas exchange (i.e. to minimise hypoxaemia and hypercapnia). Doing this has major advantages, such as decreasing tissue hypoxaemia and reduction of right ventricular afterload. It will also help to minimise lung stress and strain. These features are thought to eventually result in reduced organ failure and mortality.

Veno-arterial ECMO differs from veno-venous ECMO in the sense that the return of oxygenated blood is into the arterial circulation instead of the venous circulation. This leads to several differences, such as that the oxygenation is more homogenous in veno-venous ECMO, the risk of bleeding and leg ischaemia is higher in veno-arterial ECMO, but the major advantage of unloading of the heart is only seen in veno-arterial ECMO. The fact that veno-arterial ECMO unloads the heart by returning the oxygenated blood into the arterial system is the main reason to use in the setting requiring hemodynamic support, apart from support of gas exchange.

ECMO can be cannulated centrally, in the chest, or peripherally. Both options have their advantages and disadvantages. Central ECMO had as most important downside that the chest has to be opened and left open. Main advantage is the bigger calibre of the vessels and decreased mixing of oxygenated and de-oxygenated blood caused by counterflow, though delivery of blood in the aortic arch instead of through the groin, which is less in line with the usual physiological situation, potentially compromises oxygen delivery in the aortic arch and centrally. The size of the canullas that are indicated, also depend on the indication for which ECMO is applied. For patients in circulatory failure high flow is required and larger cannulas are indicated compared to patients with "only" hypercapnic respiratory failure.

Complications of ECMO are frequent; circuit problems (25\%), bleeding (24\%), acute kidney injury (21\%), infections (11\%) being most frequent and neurological (5\%) being most severe [13]. Meanwhile, further 
respiratory complications can be prevented, by facilitating lung protective ventilation. In ARDS, driving pressure was shown to be the most important determinant of mortality [14].

Evidence for use of ECMO for severe ARDS is still scarce and largely obtained from retrospective series. In the Conventional ventilatory support versus Extracorporeal membrane oxygenation for Severe Adult Respiratory failure (CESAR) trial patients with acute respiratory failure were referred to a specialised centre and were randomised to either the conventional arm with standard treatment protocol or the intervention arm consisting of standard protocol including consideration for ECMO. Of the patients assigned to the intervention arm, 75\% of the patients received ECMO. In the intervention group, lung protective ventilation could more often be applied and patients more often received corticosteroids. Overall, this resulted in improved survival in the group were ECMO was considered [15].

In a large multicentre randomised controlled trial, patients with severe ARDS on mechanical ventilation were randomised to either the control arm with EXPRESS ventilation (high positive end expiratory pressure, high recruitment, from the Positive End-Expiratory Pressure Setting in Adults With Acute Lung Injury and Acute Respiratory Distress Syndrome (EXPRESS) trial) or ECMO with highly protective ventilation. Importantly, there was a possibility for cross-over to ECMO for patients failing on therapy in the control group. The primary outcome of 60 -day mortality was $35 \%$ in the ECMO group compared with $46 \%$ in the control group. Although this result seems to indicate a clear benefit for ECMO, the difference was non-significant. Importantly though, rescue cross-over to ECMO occurred in $28 \%$ of patients in the control group, of whom $43 \%$ survived [16]. It was hypothesised that ECMO nonetheless has a survival benefit. In recent recommendations, ECMO is incorporated for patients with ARDS with a arterial oxygen tension $\left(P_{\mathrm{aO}_{2}}\right): F_{\mathrm{iO}_{2}}$ ratio $<150 \mathrm{mmHg}$, after other treatment options have first been considered [17] or patients with $P_{\mathrm{aO}_{2}}: F_{\mathrm{iO}_{2}}$ ratio $<80 \mathrm{mmHg}$ [18]. Currently, the The Respiratory Extracorporeal Membrane Oxygenation Survival Prediction (RESP) score is the best prediction score for survival with ECMO in ARDS [19].

A relatively new application for ECMO is bridging patients with respiratory failure to lung transplantation. Like the application of ECMO for ARDS, the practice of bridging patients to lung transplantation is increasing. Treatment decisions regarding ECMO are based on the underlying condition for which bridging will be used. Patients may suffer from pulmonary hypertension and right heart failure, necessitating choice for veno-arterial ECMO over veno-venous ECMO. It is more likely that patients with pulmonary hypertension either group 1 or group 3 listed for lung transplantation will be bridged using veno-arterial ECMO [20]. An important aspect in bridging patients to lung transplantation, differing from application of ECMO for other forms of acute respiratory failure, is the aim to ambulate patients while on ECMO. The successful ambulation of patients is even regarded as a criterium before proceeding to transplantation by some [20]. Outcomes of patients bridged with ECMO versus those not bridged are increasingly similar [21] with improving technology and increasing experience. Early complications such as primary graft failure, bleeding and acute renal failure are more common in patients bridged with ECMO [22]. Likely, centre volume is contributing to the success of bridging as experience is of key importance.

Just as important is good recipient selection, which is more strict than for non-bridged patients. Important contra-indications for bridging are irreversible extrapulmonary organ failure and heparin induced thrombocytopenia. Prolonged mechanical ventilation, increasing age and obesity are among important relative contra-indications. Pre-existing condition and the potential for ambulation play an important role whether to bridge to transplantation. Candidate selection and timing are of key importance for outcome, together with centre experience and volume. Nonetheless, not all patients can be successfully bridged to lung transplantation, also difficult decisions regarding delisting and end-off life are important.

\section{Highlights}

- ECMO can be considered in ARDS after optimal conservative management.

- ECMO is increasingly used as a bridge to transplantation in carefully selected patients.

\section{Lung transplantation ( $L T X$ ) in the era of new drugs}

This session focussed on the impact of new drugs on the indications and outcomes of lung transplantation regarding cystic fibrosis, interstitial lung diseases (ILD) and pulmonary hypertension.

First, Christian Benden pointed out that lung transplantation is still the standard of care in advanced cystic fibrosis ranging as third most frequent indication for LTx in adults and as most important indication in children [23]. According to the registry of the International Society for Heart and Lung Transplantation (ISHLT) median survival after LTx for cystic fibrosis is 9.9 years and cystic fibrosis is the subgroup with on average the best outcomes after LTx. Interestingly the best survival was seen in the $\geqslant 40$ 
years group (median survival was 10 years) and the worst one in $0-17$ years age group (median survival was 5.6 years) [24]. High mortality rate in the paediatric group was attributed to the poor adherence to the treatment, especially in teenagers [24].

As the underlying pathology of cystic fibrosis is related to the various mutations that reduce cystic fibrosis transmembrane conductance regulator (CFTR) protein function, modulators that either potentiate or correct the defect have been developed [25]. Combinations of drugs from the two groups are used, and lately a triple combination of elexacaftor/tezacaftor/ivacaftor is used in patients with PheF508del homozygous and other heterozygous mutations. The majority of cystic fibrosis patients was shown to be eligible for CFTR modulators which could restore a significant portion of the CFTR function [26]. Randomised controlled trials and open-label studies showed that cystic fibrosis patients with advanced disease could also benefit from modulator therapy [26]. Thus a larger proportion of cystic fibrosis patients will be treated with these therapies and the age of cystic fibrosis patients at transplant referral will be higher in the next year as well as the drug related toxicity issues. Lung transplantation is the ultimate therapy option for progressive lung disease if there is no other available therapy, suggesting that patients should undergo a treatment trial if new drugs become available and lastly any cystic fibrosis patient should be evaluated for transplantation if eligible [27].

Second, Sara Tomassetti emphasised that the new antifibrotic drugs have changed dramatically the clinical practice and enabled the shift from immunosuppressives to antifibrotics [28, 29]. It was shown that the new drugs not only slow down the progression of the disease, but also improve survival and reduce acute exacerbations and hospitalisations of the patients. More recently, it has also become clear that antifibrotics are effective even in other progressive fibrosing interstitial lung diseases (PF-ILD) [30, 31].

Whereas antifibrotics for idiopathic pulmonary fibrosis (IPF) are now established, several areas of uncertainty however remain for antifibrotics for PF-ILD. Long-term outcome data on survival and hospitalisation are still lacking for antifibrotics in PF-ILD. Also, many questions remain regarding the positioning of antifibrotics versus immunosuppressive treatment with respect to effect, timing and need for combination of therapy. Lastly, biomarkers for identification of treatment responders are currently still lacking.

The benefit for lung transplantation in IPF in terms of survival was demonstrated on older studies with patients with severe disease [32]. These studies showed a significant survival benefit with LTx. Whether this remains valid in the current era of antifibrotic usage needs further investigation.

Regarding IPF there are several challenges for transplantation like the presence of many comorbidities, in general advanced age and potential underlying telomerase mutations that are associated with increased complications after LTx [33]. Also the optimal timing for LTx referral, screening and listing is not always easy to be determined. New scoring systems, novel biomarkers and deep learning with artificial intelligence in the future can be used to stratify ILD patients according to prognosis and identify the subgroup that can benefit the most from LTx.

Nowadays, two approaches are used, either referral of all suitable patients at time of diagnosis particularly individuals at age limit $(>60)$ or identification of the patients that can progress rapidly (10\% decline in forced vital capacity or $15 \%$ in diffusing capacity of the lung for carbon monoxide over 6 months period, low diffusion capacity, desaturation in 6-min walk test, pulmonary hypertension, hospitalisations for respiratory deterioration) and early referral for transplantation [33].

In scleroderma, even though 1- and 5-year post-transplant survival is similar to IPF, only a limited number of patients is referred for LTx and transplanted. LTx in these patients is yet more complicated due to extrapulmonary involvement (such as oesophageal dysfunction and cardiac involvement) and the potential of relapse of the disease after LTx [34]. In the light of the current evidence it can be concluded that antifibrotics are safe drugs that should be used to attenuate the disease until transplantation and do not result in more surgical complications thus functioning as a bridge to and not a replacement of the lung transplantation [35, 36]. Novel therapies are still warranted to stabilise the disease and to overcome the need for LTx.

In the third presentation of this session, Marion Delcroix discussed the place of LTx in the treatment of pulmonary arterial hypertension (PAH), tools for timely referral and the outcomes of LTx in pulmonary hypertension. She showed that patients of PAH had 3 year survival of $73 \%$, with annual mortality of $10 \%$ in idiopathic pulmonary arterial hypertension (IPAH) patients $[37,38]$. Based on the ISHLT Registry data, the 3-year survival post-LTx in IPAH group is 64\% [39]. It is, therefore, crucial to determine adequate timing for LTx in these patients.

As per European Society of Cardiology (ESC)/ERS 2015 guidelines, LTx should be considered if there is inadequate clinical response despite double or triple sequential combination therapies [40]. As per 6th World Symposium on Pulmonary Hypertension proceedings, in non-vasoreactive and treatment-naïve 
patients at high risk, initial combination therapy including intravenous prostacyclins (PCA) is recommended and referral for LTx should also be considered [41]. The importance of risk stratification into low, intermediate and high risk was emphasised, in order to identify which subset of patients may benefit most from LTx while minimising overall risk and resource utilisation [38, 42, 43].

The utility of using REVEAL (Registry to Evaluate Early And Long-term PAH Disease Management) 2.0 scoring in risk stratification provides better characterisation of actual risk of mortality at 12 months than COMPERA (Comparative, Prospective Registry of Newly Initiated Therapies for Pulmonary Hypertension) or the French registry [44]. REVEAL 2.0 includes nonmodifiable risk factors, more variables and weighting of these variables. The frequently used lung allocation score (LAS) doesn't always adequately reflect disease severity in PAH as shown by discrepancy in observed and predicted mortality by LAS. In the REVEAL Registry, observed 1-year mortality exceeded that predicted by LAS in two subgroups of patients: those with mean right atrial pressure $\geqslant 14 \mathrm{mmHg}$ and those with 6 -MWT $\leqslant 300 \mathrm{~m}$ [45], factors that are not incorporated in the LAS. A modified LAS model that includes both 6 MWT and mean right atrial pressure better discriminates waiting list urgency for patients with PAH than the current LAS.

The study by BROUCKAERT et al. [46], a 24-year single-centre retrospective study demonstrates that bilateral LTx (BLTx) remains preferred procedure for all forms of precapillary pulmonary hypertension, except in patients with complex congenital heart disease where heart-lung transplantation (HLTx) is preferred. Overall graft and chronic lung allograft dysfunction (CLAD)-free survival were comparable and improved over time. Giant pulmonary artery aneurysm (PAA) is a late complication of IPAH. BLTx with aneurysmal repair is possible in such cases with replacing recipient PAA with donor pulmonary artery or aorta [47, 49]. HLTx remains an option for IPAH complicated by giant-sized PAA and right heart failure.

The role of ECLS as a bridge to LTx in the form of peripheral veno-arterial ECMO and NOVALUNG (a pumpless membrane oxygenator inserted between PA-LA) was highlighted, emphasising that centres performing LTx in PAH should have an established ECLS programme. In the review by HoEPER et al. [50], it was shown that 77 out of 81 patients received ECMO as a bridge to transplant. 72 out of 77 patients (94\%) were successfully bridged to transplant with $78 \%$ of them getting discharged. Intraoperative ECMO versus cardiopulmonary bypass $(\mathrm{CPB})$ was highlighted with reference to study by DeLL'Amore et al. [51], stating that intraoperative ECMO has almost completely replaced CPB as it is associated with reduced peri-operative complications including renal failure, reduced need of transfusions of blood products and better survival. In patients with pulmonary hypertension and right-heart failure, veno-arterial ECMO is occasionally established prior to general anesthesia to avoid haemodynamic instability. Indications for CPB remain: extreme cardiomegaly which makes the access to the left pulmonary hilum without complete heart emptying impossible, severe dilatation of the main branches of the pulmonary arteries and supra-systemic pulmonary artery pressure with reduced cardiac index.

BLTx for IPAH with intraoperative veno-arterial ECMO support seems to provide superior outcome compared with the results reported about the use of CPB. Prophylactic prolongation of veno-arterial ECMO into the early postoperative period provides stable postoperative conditions and better survival outcomes [52]. The main cause of primary graft dysfunction (PGD) in this patient group is usually not residual pulmonary hypertension but incapability of the left ventricle to cope with the preload after LTx. It was demonstrated in this study that an important problem causing early PGD after BLTx in patients with end-stage PAH is, rather than elevated pulmonary pressures with increased shear stress or right ventricle failure, left ventricle dysfunction with predominantly diastolic failure, and elevated filling pressures. The above study stated that early after BLTx for severe pulmonary hypertension, the left ventricle may be unable to handle normalised left ventricle preload. This can be effectively bridged with awake veno-arterial ECMO [53]. Also, at $72 \mathrm{~h}$, the PGD scores in the BLTx-ECMO and HLTx groups were significantly lower than those in the BLTx-ventilation group.

In the final presentation, the aim by Jens Gottlieb was to describe the effects of the new therapeutic strategies in patients with pulmonary hypertension, ILD and cystic fibrosis on needs and indications for LTx and to evaluate outcomes of LTx for the three indications. Based on the ISHLT Registry data, ILD is increasing as the primary indication for LTx, whilst cystic fibrosis has remained the same in last few years [39]. The ILD subgroup patients comprise a relatively elderly cohort with multiple co-morbidities while the cystic fibrosis cohort comprises a relatively younger population. The Collaborative Transplant Study (CTS) Registry data for LTx shows that survival improved post LTx from 42.6\% 5-year survival (before 2000) to $56.5 \%$ 5-year survival (after 2000). The ISHLT Registry shows that conditional 1-year survival was best in cystic fibrosis subgroup (85\%), followed by IPF (80\%) and worst in PAH (74\%) [39]. However, comparing the conditional 5-year survival data, the PAH subgroup had the best long-term survival (75\%) along with cystic fibrosis (75\%), while IPF had worst survival (64\%). Above the age of 65 years the 5-year survival was only $38 \%$. 
Association of transplant centre volume and post-LTx mortality was discussed [54]. Lower transplant volume centre is independently associated with higher 5-year mortality. Many retrospective studies were analysed with specific mention of studies by VocK et al. [55] and THABUT et al. [56], concluding that there is survival benefit post-LTx, with the greatest potential benefit in patients with higher lung allocation scores or restrictive native lung disease or cystic fibrosis. Thabut et al. [56] showed a median survival of 8.9 years for cystic fibrosis patients, 6.7 years for COPD with $\alpha_{1}$ antitrypsin deficiency (AATD), 5.6 years for COPD without AATD, 4.8 years for idiopathic interstitial pneumonia and 2.8 years for re-transplantation.

In the first year post-LTx, the common causes of death were infections and graft failure while after the first year, the important causes of death are CLAD followed by infections, malignancy, etc. Recipient age was more important than the donor age [39]. Within 5 years post-LTx, hypertension $(81.7 \%)$ was the most common morbidity followed by renal dysfunction (53.9\%), CLAD (41.6\%) and diabetes mellitus (35.9\%) [57].

The new definition of CLAD was recently published by VERLEDEN et al. [58], and defined as a substantial and persistent decline $(\geqslant 20 \%)$ in measured forced expiratory volume in 1 s $\left(\mathrm{FEV}_{1}\right)$ value from the reference (baseline) value. Two important phenotypes of CLAD exist: obstructive and restrictive CLAD. The obstructive (bronchiolitis obliterans syndrome (BOS)) phenotype has a better survival than restrictive (restrictive allograft syndrome (RAS)) phenotype. BOS had 5-year survival of $46 \%$ while RAS phenotype had $0 \% 5$-year survival.

In a study by SINGER et al. [59] it was shown that LTx improved Health Related Quality of Life (HRQoL) by all five measures $(\mathrm{p}<0.05)$ and all but Short Form $(\mathrm{SF}) 12-$ Mental Health improved by three-fold or greater than the minimally clinically important difference. Visual analogue scale (VAS) quality of life (QoL) showed an improvement from 40 pre-transplant to 80 in post-transplant. Older subjects ( $\geqslant 65$ years) had substantially smaller improvement in QoL. Smallest benefit was seen in PAH subgroup. Patients age 65 years or older derived substantially less HRQoL benefit than younger patients with $11 \%$ in study not improving in QoL. A second study by SINGER et al. [60] showed that age was not associated with meaningful differences in the HRQoL benefits of transplantation. There was less HRQoL benefit in ILD than in cystic fibrosis. Regarding return to work post-LTx, according to the ISHLT Registry, $14 \%$ of patients returned to work at 1 year with only $18 \%$ at 5 years [39]. In another study by SuHLING et al., [61], $38 \%$ of the patients were employed after LTx. Employment was associated with a better QoL and median time from transplantation to the return to work was 11 months. In a Swiss study of LTx in cystic fibrosis recipients by RADTKE et al., [62] the number of subjects employed at 3-5 years post LTx was 75\%.

\section{Highlights}

- The majority of cystic fibrosis patients is eligible for CFTR modulator therapy and a treatment trial is indicated prior to lung transplantation.

- Novel antifibrotics are effective to slow down progression in IPF and PF-ILD and can be safely used until the time of transplantation.

- There is a crucial role of ECMO before, during and after LTx for PAH.

- LTx is associated with significant increase in QoL, but less so in elderly recipients.

\section{Conclusions}

Due to the coronavirus disease 2019 pandemic, this congress was the first "Virtual" International Congress of the ERS and it was a great success, with very diverse and important sessions on innovation and state of the art in thoracic surgery and lung transplantation. In this article, we summarised the highlights of the most important sessions of this congress representing a wide range of topics. We look forward to next year's conference, to be held in Barcelona, Spain, September 4-8, 2021.

Conflict of interest: D. Esendagli has nothing to disclose. U. Shah has nothing to disclose. G. Batihan has nothing to disclose. D. Magouliotis has nothing to disclose. F. Meloni has nothing to disclose. R. Vos is a Senior Clinical Research Fellow of the Research Foundation Flanders (FWO); his institution has received research grants but he has no conflicts of interest to report regarding the current manuscript. S. Elia has nothing to disclose. M. Hellemons has nothing to disclose.

\section{References}

1 Veronesi G, Galetta D, Maisonneuve P, et al. Four-arm robotic lobectomy for the treatment of early-stage lung cancer. J Thorac Cardiovasc Surg 2010; 140: 19-25.

2 Lee EC, Lazzaro RS, Glassman LR, et al. Switching from thoracoscopic to robotic platform for lobectomy: report of learning curve and outcome. Innovations (Phila) 2020; 15: 235-242. 
3 Veronesi G, Park B, Cerfolio R, et al. Robotic resection of Stage III lung cancer: an international retrospective study. Eur J Cardiothorac Surg 2018; 54: 912-919.

4 Solinas M, Novellis P, Bottoni E, et al. Robotic approach in case of thymoma involving the left anonymous vein: a case report. AME Case Rep 2017; 1: 3.

5 Kanzaki M. Current status of robot-assisted thoracoscopic surgery for lung cancer. Surg Today 2019; 49: 795-802.

6 Guo F, Ma D, Li S. Compare the prognosis of Da Vinci robot-assisted thoracic surgery (RATS) with video-assisted thoracic surgery (VATS) for non-small cell lung cancer: A Meta-analysis. Medicine (Baltimore) 2019; 98 : e17089.

$7 \quad$ Larici AR, Farchione A, Franchi P, et al. Lung nodules: size still matters. Eur Respir Rev 2017; 26 : 170025.

8 Naidich DP, Bankier AA, MacMahon H, et al. Recommendations for the management of subsolid pulmonary nodules detected at CT: a statement from the Fleischner Society. Radiology 2013; 266: 304-317.

9 Song Q, Zhao L, Luo X, et al. Using deep learning for classification of lung nodules on computed tomography images. J Healthc Eng 2017; 2017: 8314740.

10 Kodama H, Yamakado K, Hasegawa T, et al. Radiofrequency ablation for ground-glass opacity-dominant lung adenocarcinoma. J Vasc Interv Radiol 2014; 25: 333-339.

11 Onishi H, Shioyama Y, Matsumoto Y, et al. Stereotactic body radiotherapy in patients with lung tumors composed of mainly ground-glass opacity. J Radiat Res 2020; 61: 426-430.

12 Lorusso R, Alexander P, Rycus P, et al. The extracorporeal life support organization registry: update and perspectives. Ann Cardiothorac Surg 2019; 8: 93-98.

13 Brodie D, Slutsky AS, Combes A. Extracorporeal life support for adults with respiratory failure and related indications: a review. JAMA 2019; 322: 557-568.

14 Neto AS, Schultz MJ, Festic E. Ventilatory support of patients with sepsis or septic shock in resource-limited settings. Intensive Care Med 2016; 42: 100-103.

15 Peek GJ, Mugford M, Tiruvoipati R, et al. Efficacy and economic assessment of conventional ventilatory support versus extracorporeal membrane oxygenation for severe adult respiratory failure (CESAR): a multicentre randomised controlled trial. Lancet 2009; 374: 1351-1363.

16 Combes A, Hajage D, Capellier G, et al. Extracorporeal membrane oxygenation for severe acute respiratory distress syndrome. N Engl J Med 2018; 378: 1965-1975.

17 Abrams D, Ferguson ND, Brochard L, et al. ECMO for ARDS: from salvage to standard of care? Lancet Respir Med 2019; 7: 108-110.

18 Papazian L, Aubron C, Brochard L, et al. Formal guidelines: management of acute respiratory distress syndrome. Ann Intensive Care 2019; 9: 69.

19 Schmidt M, Bailey M, Sheldrake J, et al. Predicting survival after extracorporeal membrane oxygenation for severe acute respiratory failure. The Respiratory Extracorporeal Membrane Oxygenation Survival Prediction (RESP) score. Am J Respir Crit Care Med 2014; 189: 1374-1382.

20 Tipograf Y, Salna M, Minko E, et al. Outcomes of extracorporeal membrane oxygenation as a bridge to lung transplantation. Ann Thorac Surg 2019; 107: 1456-1463.

21 Benazzo A, Schwarz S, Frommlet F, et al. Twenty-year experience with extracorporeal life support as bridge to lung transplantation. J Thorac Cardiovasc Surg 2019; 157: 2515-2525.

22 Ius F, Natanov R, Salman J, et al. Extracorporeal membrane oxygenation as a bridge to lung transplantation may not impact overall mortality risk after transplantation: results from a 7 -year single-centre experience. Eur $J$ Cardiothorac Surg 2018; 54: 334-340.

23 Benden C. Lung transplantation as standard of care for advanced cystic fibrosis lung disease. J Heart Lung Transplant 2020; 39: 561-562.

24 Benden C, Goldfarb SB, Stehlik J. An aging population of patients with cystic fibrosis undergoes lung transplantation: An analysis of the ISHLT Thoracic Transplant Registry. J Heart Lung Transplant 2019; 38: $1162-1169$.

25 Derichs N. Targeting a genetic defect: cystic fibrosis transmembrane conductance regulator modulators in cystic fibrosis. Eur Respir Rev 2013; 22: 58-65.

26 Shteinberg M, Taylor-Cousar JL. Impact of CFTR modulator use on outcomes in people with severe cystic fibrosis lung disease. Eur Respir Rev. 2020; 29: 190112.

27 Weill D, Benden C, Corris PA, et al. A consensus document for the selection of lung transplant candidates: 2014-an update from the Pulmonary Transplantation Council of the International Society for Heart and Lung Transplantation. J Heart Lung Transplant 2015; 34: 1-15.

28 King TE Jr, Bradford WZ, Castro-Bernardini S, et al. A phase 3 trial of pirfenidone in patients with idiopathic pulmonary fibrosis. N Engl J Med 2014; 370: 2083-2092.

29 Richeldi L, Du Bois RM, Raghu G, et al. Efficacy and safety of nintedanib in idiopathic pulmonary fibrosis. $N$ Engl J Med 2014; 370: 2071-2082.

30 Flaherty KR, Wells AU, Cottin V, et al. Nintedanib in progressive fibrosing interstitial lung diseases. $N$ Engl J Med 2019; 381: 1718-1727.

31 Distler O, Highland KB, Gahlemann M, et al. Nintedanib for systemic sclerosis-associated interstitial lung disease. N Engl J Med 2019; 380: 2518-2528.

32 Thabut G, Mal H, Castier Y, et al. Survival benefit of lung transplantation for patients with idiopathic pulmonary fibrosis. J Thorac Cardiovasc Surg 2003; 126: 469-475.

33 George PM, Patterson CM, Reed AK, et al. Lung transplantation for idiopathic pulmonary fibrosis. Lancet Respir Med 2019; 7: 271-282.

34 Mackintosh JA, Stainer A, Barnett JL, et al. Systemic Sclerosis Associated Interstitial Lung Disease: A Comprehensive Overview. Seminars in respiratory and critical care medicine 2019; 40: 208-226.

35 Leuschner G, Stocker F, Veit T, et al. Outcome of lung transplantation in idiopathic pulmonary fibrosis with previous anti-fibrotic therapy. J Heart Lung Transp 2018; 37: 268-274.

36 Delanote I, Wuyts WA, Yserbyt J, et al. Safety and efficacy of bridging to lung transplantation with antifibrotic drugs in idiopathic pulmonary fibrosis: a case series. BMC Pulm Med 2016; 16: 156.

37 Humbert M, Sitbon O, Chaouat A, et al. Survival in patients with idiopathic, familial, and anorexigen-associated pulmonary arterial hypertension in the modern management era. Circulation 2010; 122: 156-163. 
Boucly A, Weatherald J, Savale L, et al. Risk assessm
arterial hypertension. Eur Respir J 2017; 50: 1700889.

Chambers DC, Cherikh WS, Harhay MO, et al. The International Thoracic Organ Transplant Registry of the International Society for Heart and Lung Transplantation: Thirty-sixth adult lung and heart-lung transplantation Report-2019; Focus theme: Donor and recipient size match. J Heart Lung Transplant 2019; 38: $1042-1055$.

40 Galie N, Humbert M, Vachiery JL, et al. 2015 ESC/ERS Guidelines for the diagnosis and treatment of pulmonary hypertension. Eur Respir J 2015; 46: 903-975.

41 Galiè N, Channick RN, Frantz RP, et al. Risk stratification and medical therapy of pulmonary arterial hypertension. Eur Respir J 2019; 53: 1801889.

42 Kylhammar D, Kjellström B, Hjalmarsson C, et al. A comprehensive risk stratification at early follow-up determines prognosis in pulmonary arterial hypertension. Eur Heart J 2018; 39: 4175-4181.

43 Hoeper MM, Kramer T, Pan Z, et al. Mortality in pulmonary arterial hypertension: prediction by the 2015 European pulmonary hypertension guidelines risk stratification model. Eur Respir J 2017; 50: 1700740.

44 Benza RL, Gomberg-Maitland M, Elliott CG, et al. Predicting survival in patients with pulmonary arterial hypertension: The REVEAL risk score calculator 2.0 and comparison with ESC/ERS-based risk assessment strategies. Chest 2019; 156: 323-337.

45 Benza RL, Miller DP, Frost A, et al. Analysis of the lung allocation score estimation of risk of death in patients with pulmonary arterial hypertension using data from the REVEAL Registry. Transplantation 2010; 90: $298-305$.

46 Brouckaert J, Verleden SE, Verbelen T, et al. Doublelung versus heartlung transplantation for precapillary pulmonary arterial hypertension: a 24year singlecenter retrospective study. Transpl Int 2019; 32: 717-729.

47 Eadington T, Santhanakrishnan K, Venkateswaran R. Heart-lung transplantation for idiopathic pulmonary arterial hypertension and giant pulmonary artery aneurysm - case report. J Cardiothorac Surg 2020; 15: 169.

48 Schwarz S, Benazzo A, Prosch H, et al. Lung transplantation for pulmonary hypertension with giant pulmonary artery aneurysm. J Thorac Cardiovasc Surg 2020; 159: 2543-2550.

49 Egan TMIkonomidis J. Commentary: Is there more than one way to skin a pulmonary artery aneurysm? J Thorac Cardiovasc Surg 2020; 159: 2553-2554.

50 Hoeper MM, Benza RL, Corris P, et al. Intensive care, right ventricular support and lung transplantation in patients with pulmonary hypertension. Eur Respir J 2019; 53: 1801906.

51 Dell'Amore A, Campisi A, Congiu S, et al. Extracorporeal life support during and after bilateral sequential lung transplantation in patients with pulmonary artery hypertension. Artif Organs 2020; 44(6): 628-637.

52 Moser B, Jaksch P, Taghavi S, et al. Lung transplantation for idiopathic pulmonary arterial hypertension on intraoperative and postoperatively prolonged extracorporeal membrane oxygenation provides optimally controlled reperfusion and excellent outcome. Eur I Cardiothorac Surg 2018; 53(1): 178-185.

53 Tudorache I, Sommer W, Kühn C, et al. Lung transplantation for severe pulmonary hypertension--awake extracorporeal membrane oxygenation for postoperative left ventricular remodelling. Transplantation 2015; 99: 451-458.

54 Chambers DC, Cherikh WS, Goldfarb SB, et al. The International Thoracic Organ Transplant Registry of the International Society for Heart and Lung Transplantation: Thirty-Fifth Adult Lung and Heart-Lung Transplant Report-2018; focus theme: multiorgan transplantation. J Heart Lung Transplant 2018; 37: 1169-1118.

55 Vock DM, Durheim MT, Tsuang WM, et al. Survival benefit of lung transplantation in the modern era of lung allocation. Ann Am Thorac Soc 2017; 14: 172-181.

56 Thabut G, Mal H. Outcomes after lung transplantation. I Thorac Dis 2017; 9: 2684-2691.

57 Yusen RD, Edwards LB, Dipchand AI, et al. The Registry of the International Society for Heart and Lung Transplantation: Thirty-third Adult Lung and Heart-Lung Transplant Report-2016; focus theme: Primary diagnostic indications for transplant. J Heart Lung Transplant 2016; 35: 1170-1184.

58 Verleden GM, Glanville AR, Lease ED, et al. Chronic lung allograft dysfunction: Definition, diagnostic criteria, and approaches to treatment-A consensus report from the Pulmonary Council of the ISHLT. J Heart Lung Transplant 2019; 38: 493-503

59 Singer JP, Katz PP, Soong A, et al. Effect of lung transplantation on health-related quality of life in the era of the lung allocation score: a U.S. prospective cohort study. Am J Transplant 2017; 17: 1334-1345.

60 Singer LG, Chowdhury NA, Faughnan ME, et al. Effects of recipient age and diagnosis on health-related quality-of-life benefit of lung transplantation. Am J Respir Crit Care Med 2015; 192: 965-973.

61 Suhling H, Knuth C, Haverich A, et al. Employment after lung transplantation-a single-center cross-sectional study. Deutsches Ärzteblatt Int 2015; 112: 213.

62 Radtke T, Königs A, Chen X, et al. Predictors of long-term employment among patients with cystic fibrosis undergoing lung transplantation. Swiss Med Wkly 2020; 150: w20286. 